\title{
Algorithm for a high precision contactless measurement system
}

\author{
Tran Trung Nguyen, Arvid Amthor, Christoph Ament \\ System Analysis Group, Ilmenau University of Technology, P.O. Box 100565, 98684 IImenau, Germany
}

\section{ABSTRACT}

This paper presents a self-calibration method for the multi laser tracker system (MLTS) which can track a retroreflector mounted on a kinematical system (e.g. positioning stage, robot manipulator etc.). Four laser trackers build up the MLTS. In the first part of the study the required algorithms enabling the MLTS to measure the position of the retroreflector are presented. The algorithms include the localization of the retroreflector, the communication between the laser trackers and the tracking controller as well as the calculation of the Tool Centre Point (TCP) position. In the second part of this study a detailed analysis of the high precision self-calibration algorithm is carried out. A sensitivity analysis shows that the quality of the parameter estimation highly depends on the optimal arrangement of the MTLS.

Section: RESEARCH PAPER

Keywords: optical measurement, multi laser tracker system, self-calibration

Citation: Tran Trung Nguyen, Arvid Amthor, Christoph Ament, Algorithm for a high precision contactless measurement system, Acta IMEKO, vol. 2, no. 2, article 3, December 2013, identifier: IMEKO-ACTA-02 (2013)-02-03

Editor: Paolo Carbone, University of Perugia

Received February $19^{\text {th }}, 2013$; In final form September $9^{\text {th }}, 2013$; Published December 2013

Copyright: (C) 2013 IMEKO. This is an open-access article distributed under the terms of the Creative Commons Attribution 3.0 License, which permits unrestricted use, distribution, and reproduction in any medium, provided the original author and source are credited

Funding: This work was done in the framework of the project "Kompetenzdreieck Optische Mikrosysteme - Spitzenforschung und Innovation in den neuen Ländern", which is supported by the German Ministry of Education and Research (FKZ: 16SV5473).

Corresponding author: Tran Trung Nguyen, e-mail: tran-trung.nguyen@tu-ilmenau.de

\section{INTRODUCTION}

The accurate position of the Tool Centre Point (TCP) during the production process is a crucial issue for efficient machine processing of complex tasks with smaller and smaller components and structures of steadily decreasing size. Capacitive and inductive position sensors achieve a limited measurement resolution and/or a limited working range and need to be integrated in the process machine. As the sensors measure the TCP position indirectly subsequent measurement errors are induced. Also all alignment errors of a measured serial kinematics deteriorate the measured position of the TCP. In contrast, optical sensors offer a high precision and a large working range. Apart from that, optical sensors measure the TCP position directly and do not need to be integrated in the process machine. The preferred optical measurement device especially in high precision applications is the laser tracker.

A laser tracker is an optical instrument which permits a contactless 3D measurement of a moving target. The device consists of a very precise laser interferometer and a beam deflection system.
The development of laser trackers began in the 1980's when Lau et al. [7][8] used the laser tracking technique to determine the performance of a robot. This tracking system was implemented in a real-time system to identify the three-dimensional static as well as dynamic positioning accuracy of a robot end effector. The measuring volume of the described tracking system was approximately $3 \times 3 \times 3 \mathrm{~m}$ and a maximum speed of 300 $\mathrm{mm} / \mathrm{s}$ was reached. In order to enhance the tracking speed Parker and Mayer developed an optical laser tracking system using a 2-axis rotational galvanometer scanner as beam deflection unit [14][15][16]. Their system was used to measure the absolute position of a moving optical target which was mounted on a robot's manipulator. The first who utilized a laser interferometer for a precise distance measurement were Takatsuji et al. They presented an interferometer based laser tracking system called distance-only-measurement (DOM). This system determined the position of a tracked target using the method of trilateration and consists of four single laser tracking units. Using four systems detecting one target offers the advantage of a redundant measurement. Thus, the position of the tracker units and the initial position of the target can be calculated from 
multiple position measurements. The resulting measurement error was about 40 microns with a target distance of one meter [18].

Due to the high resolution of the interferometer the DOM method is state of the art in non-contact high precision metrology. Beside the achievable precision an additional advantage of the proposed method is the fact that the three-dimensional position of the reflector is detected directly and hence Abbe's principle is not violated. The crucial question concerning the trilateration is the determination of the absolute coordinates of the reflector and the position of each laser tracker given the initial measurement lengths. These parameters are calculated by self-calibration methods without the usage of a reference calibration device. In order to apply the self-calibration algorithm the number of free measurement points must be greater than the number of unknown parameters. This leads to an overconstrained system of equations, which can be solved using powerful numerical techniques.

The authors of this contribution found that the quality of the self-calibration algorithm depends on various metrological arrangements of the measuring system e.g. the number of measurements, the working range, the distance of the laser tracker among each other and the measurement point. In [19] an optimal arrangement of four laser trackers is clarified. Nevertheless, there is no mathematical description for the various arrangements of measurement points and the laser trackers available up to date and hence an optimal configuration for the self-calibration is not given. Numerical simulations can (or will) be used to close this gap.

The present contribution is organized as follows. Within the framework of the "Kompetenzdreieck Optische Mikrosystem (OPTIMI)", which is supported by the Ministry of Education and Research (BMBF), the Systems Analysis Group of Ilmenau University of Technology has developed a MLTS for tracking a kinematical system [1][2][3][4][5][6] (see Figure 1).

The hard- and software components of this experimental set-up is presented in the following section. Section 3 describes the required tracking control algorithm of one laser tracking unit and Section 4 presents the calculation of the TCP position using the trilateration method. Finally, selected results of the parameter estimation are shown in order to increase the accuracy of the calibration method presented. The results are further concluded and a short outlook is given.

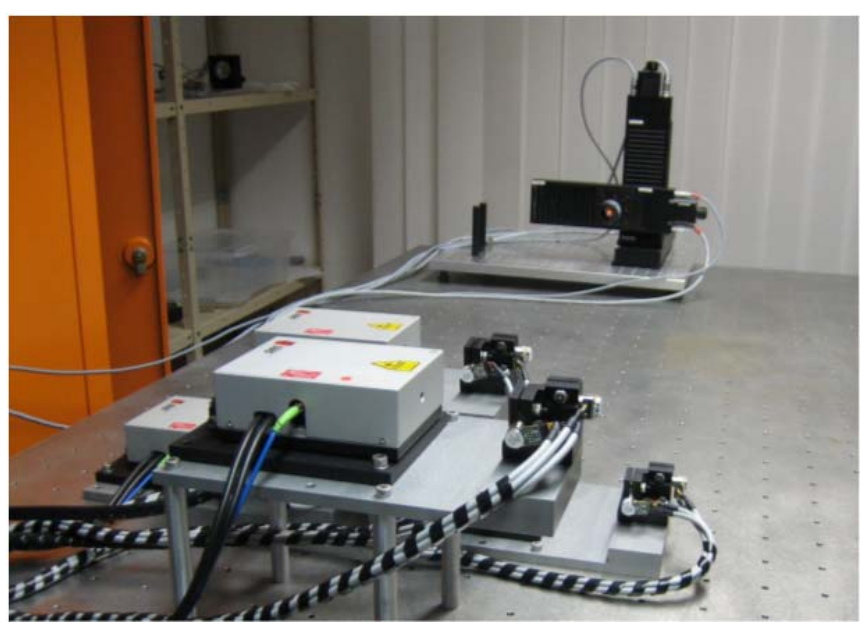

Figure 1. The developed MLTS for tracking kinematics.

\section{EXPERIMENTAL SET-UP}

The set-up of a single laser tracker module is decomposed of the opto-electronic components, the supply electronic unit and the opto-electronic detection unit (Figure 2).

The opto-electronic components include a Michelson interferometer and a galvanometer scanner. Both components are fixed on a base panel. The homodyne interferometer uses a stabilized $\mathrm{He}-\mathrm{Ne}$ laser as beam source and realizes a position resolution on sub nanometer scale [9][10]. Beside the Michelson interferometer a four quadrant diode (4QD) unit is integrated. In this study the utilized 4QD has an active area of $5 \times 5 \mathrm{~mm}$. The four quadrants are separated by a gap, which amounts to $30 \mu \mathrm{m}$. This kind of diode is able to detect a misalignment between the interferometer and the retro-reflector. A corner cube mirror is used as a retro-reflector in this work [22]. The described opto-electronic components are arranged in such a way that the emitted beam hits the galvanometer scanner. The galvanometer scanner consists of two beam deflection units and the laser beam is reflected by both of them [1]. Each deflection unit consists of a mirror, a torque transducer, a high precision angle sensor and an analogue servo closed loop control. The servo is used to control the angles and the current required for the actuators.

The developed algorithms (localization, communication, tracking control and determination of the TCP position) are implemented in Matlab/Simulink ${ }^{\circledR}$ using C-code-s-functions. The Simulink Coder ${ }^{\circledR}$ is used to automatically generate $C$ code, which is carried out by a modular Rapid Control Prototyping System of dSpace ${ }^{\circledR}$. All algorithms work with a sample rate of $10 \mathrm{kHz}$.

Figure 3 depicts the functionality of one laser tracker module in detail. The laser beam is transported to the interferometer via an optical fibre. Inside the interferometer the laser beam hits the first polarizing beam splitter B1 and dividing the beam into a reference beam and a measuring beam. The reference beam then hits the fixed reference mirror and returns to the first beam splitter B1. Simultaneously, the measuring beam passes the second beam splitter B2 and leaves the interferometer. Starting from the interferometer the measurement beam hits the first mirror of the galvanometer scanner, which is rotating around the $x$-axis. The x-galvanometer scanner (x-scanner) deflects the measurement beam to the second mirror, which is rotating around the $y$-axis. The y-galvanometer scanner (yscanner) deflects the measurement beam again to the retro-

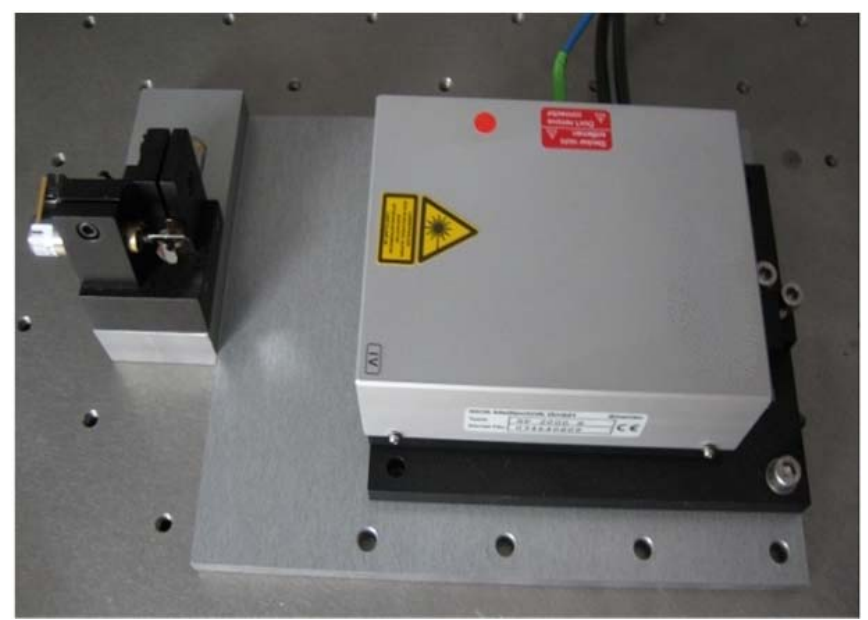

Figure 2. The developed tracker module. 


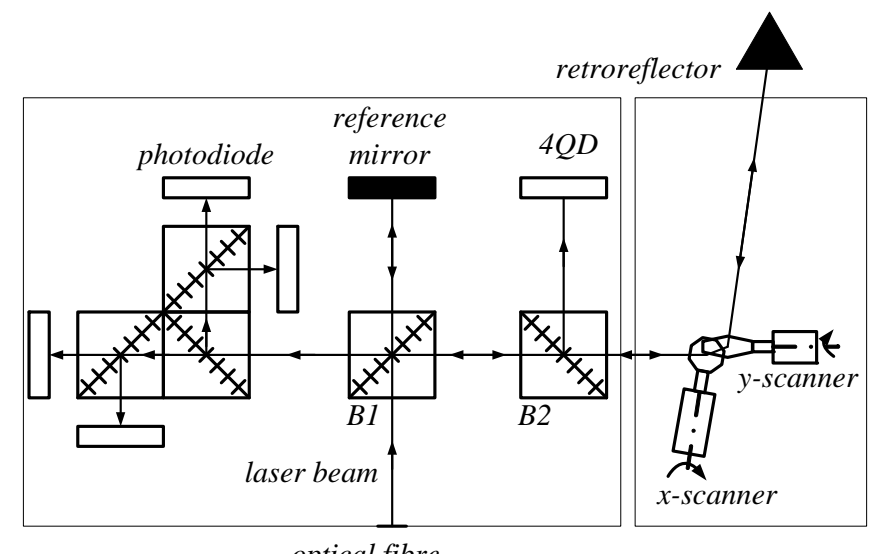

optical fibre

Figure 3. The principle of the tracker module.

reflector. The retro-reflector is fixed on the TCP of the kinematics tracked. It reflects the measurement beam back to the galvanometer scanner. The reflected beam returns to the interferometer via the galvanometer scanner and hits the second beam splitter B2. Part of the reflected beam reaches the 4QD. This signal of the 4QD is used to track the target.

The other part of the reflected beam passes through the second beam splitter B2 and is superimposed on the reference beam in the beam splitter B1. Furthermore, the superimposed beam hits the opto-electronic detection unit. A resolution of 0.1 $\mathrm{nm}$ is achieved for the position detection [10].

\section{TRACKING ALGORITHM}

\subsection{Localization of the retro-reflector}

The proposed localization method is based on an Archimedean spiral which is derived in polar coordinates. The spiral is defined by three parameters. The radius $r_{0}$ is used to describe the maximum rotational angle of the galvanometer scanner in polar coordinates. The duration $t_{0}$ defines the rotation of the laser beam from the origin of the spiral to the predefined radius $r_{0}$. The number of rotations is given by the parameter $\omega_{0}$. To describe the Archimedean spiral showed in Figure 4 the following equations in polar coordinates are given for the $x$-axis and for the $y$-axis:

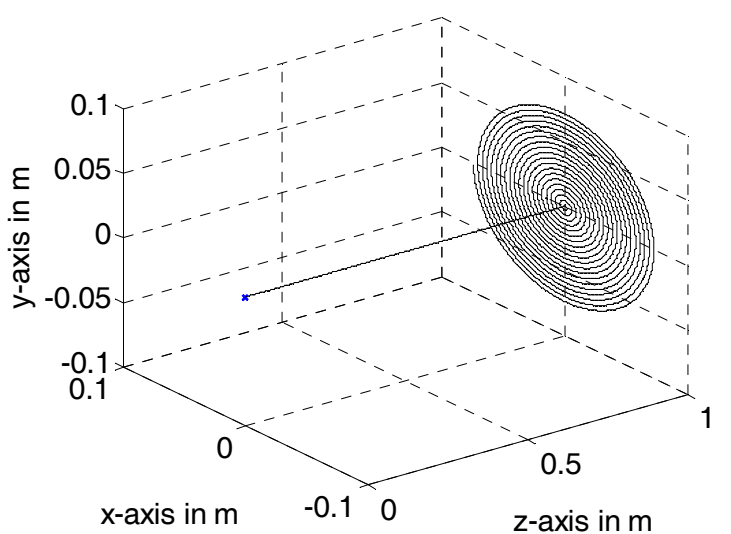

Figure 4. The designed spiral in Cartesian coordinates.

$$
\begin{aligned}
& x(t)=\frac{r_{0}}{t_{0}} t \cos \left(2 \pi \frac{\omega_{0}}{t_{0}} t\right) \\
& y(t)=\frac{r_{0}}{t_{0}} t \sin \left(2 \pi \frac{\omega_{0}}{t_{0}} t\right)
\end{aligned}
$$

Due to the fact that the behaviour of the galvanometer scanner is specified in angle coordinates, the designed Archimedean spiral has to be transformed from polar coordinates into angle coordinates. The rotation angles of the galvanometer scanner are calculated as follows:

$$
\begin{aligned}
& \theta(t)=\arctan \left(\frac{x(t)}{l}\right) \\
& \varphi(t)=\arctan \left(\frac{y(t)}{l}\right)
\end{aligned}
$$

In Equation (2) the parameter $l$ is the distance between the laser tracker and the localization plane. The distance is predefined by $1 \mathrm{~m}$. The angle $\theta(t)$ describes the rotation of the galvanometer scanner about the $x$-axis and the angle $\varphi(t)$ describes the rotation of the galvanometer scanner about the $y$-axis.

\subsection{Communication of the MLTS}

The proposed localization algorithm is used for a single laser tracker module. Due to the fact that four tracker modules are used in an MLTS, the developed algorithm is expanded by a communication channel between the four tracker modules. Hence, the tracker modules are able to share the angle information and the retroreflector position with each other and this significantly accelerates the localization (all trackers have found the TCP) of the whole MLTS. To realize an effective acceleration of the localization phase, the approximate (low precision) TCP position is required in a global coordinate system (see Section 4.2). Initially, all tracker modules are searching for the TCP. If at least two trackers find the TCP the triangulation method will be used to determine the TCP position. The TCP position is subsequently transferred to the untraced tracker modules and therefore they find the TCP immediately. It has to be mentioned that during localization the approximate position coordinates of all tracker modules are required. For this purpose a low precision calibration process based on the angles and distances estimates the coordinates of the four tracker modules. For detailed information on the TCP localization process the reader is referred to [14].

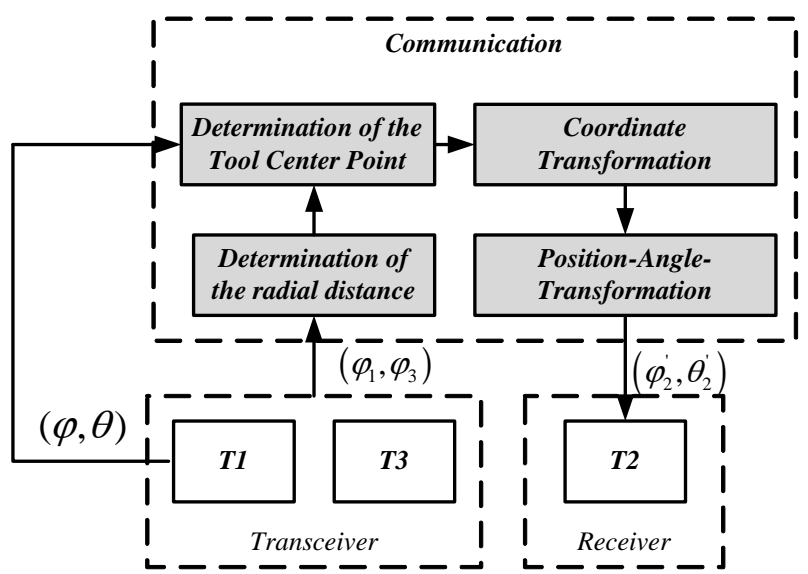

Figure 5. An example of the communication protocol. 
Figure 5 shows an example of a communication sequence. Initially, the tracker modules $T_{3}$ as well as $T_{1}$ have found the retroreflector. Based on the radial distance between the two tracker modules and the retro-reflector as well as the angle information the position of the retro-reflector is computed in the spherical coordinates of e.g. $T_{1}$. The position of the retroreflector is then transformed to the global coordinate system of MLTS. In a final step, the global TCP position is transformed to the polar coordinates of the residual (still searching) tracker modules, in our example $T_{2}$. The local position is now shared with $T_{2}$ and $T_{2}$ finds the TCP immediately.

To combine the localization algorithm with the communication of the MLTS a state machine showed in Figure 6 was designed. This finite-state machine is used to model the switch behaviour of every tracker module in operation. The algorithm has three binary input variables $(a, n, p)$ and two output variables $\left(\theta_{\infty}, \varphi_{y}\right)$.

The output variables are the rotation angles of the tracker module and the input variables are:

- $a$ : communication mode active

- $n$ : tracker does not hit the retroreflector

- $\quad p$ : localization mode active

Four finite-states $\left(Z_{00}, Z_{01}, Z_{10}\right.$ and $\left.Z_{11}\right)$ are used during the localization:

- $Z_{00}$ : The localization mode is deactivated. The tracker module has no information about the position of the retroreflector.

- $Z_{01}$ : The localization mode is activated and the communication is deactivated. The tracker module searches the retroreflector using the proposed localization algorithm.

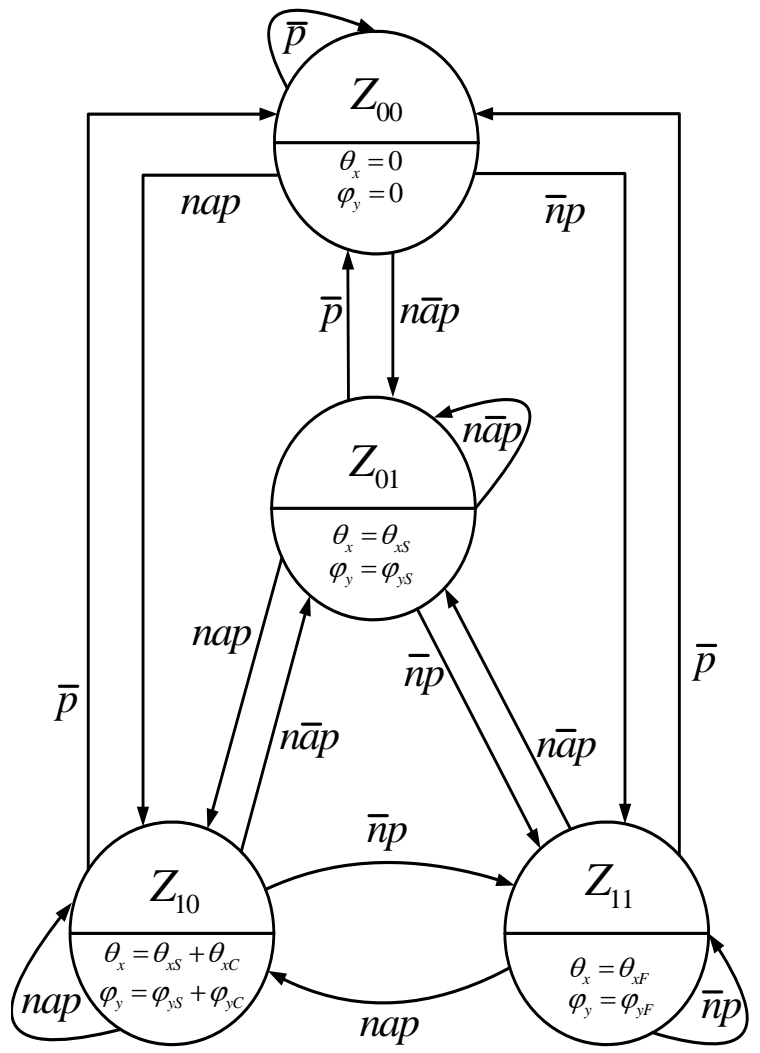

Figure 6 . The state machine describing the performance of the localization algorithm.
- $Z_{10}$ : A minimum number of two tracker modules hit the target center of the retroreflector. The communication mode is activated. Using this additional information the other tracker modules are looking for the retroreflector in a defined small area around the communicated TCP position.

- $Z_{11}$ : All tracker modules have found the retroreflector.

\subsection{Tracking control}

In the case that the laser beam has found the retroreflector, the beam has to track it. For this purpose a feedback control algorithm has to be implemented. A model based control approach is one possibility to minimize the tracking error of the laser beam. The requirements in this case are stability, large bandwidth and robustness against external disturbances.

The 4QD is used as a feedback sensor and to provide the displacement of the reflected beam as a $x_{4 Q D}$ - and $y_{4 Q D}-$ signal, respectively. If the beam hits the target center, then the laser beam is found in the origin of the 4QD coordinates. Therefore the $x_{4 O D}$-signal only affects the $\mathrm{x}$-scanner and the $y_{4 Q D}$-signal only affects the $y$-mirror. Figure 7 shows the structure of the closed loop tracking control scheme for one axis. The motion of the tracking kinematics $x_{\text {Retro }}$ in $\mathrm{x}$-direction generates a 4QD signal $\left(x_{4 Q D}\right)$, which is handled as a dynamic disturbance. The error $e$ between the set point $w_{4 Q D}$ and the disturbance signal $x_{4 Q D}$ is compensated by a PID controller, which generates an output, $\varphi_{\text {feedback }}$. The output $\varphi_{\text {dis }}$ is computed by the disturbance compensation unit $G_{D}$ in order to suppress the influence of the moving stage. The compensation unit $G_{D}$ reflects the inverse system dynamics of the galvanometer scanner $G_{M}$ and the beam path $G_{B}$. Thus, the disturbance compensation unit acts as a feed forward controller and reduces the tracking error of the laser beam, which tracks the positioning stage. The regulating variable $\varphi_{r f}$ is a combination of outputs $\varphi_{\text {feedback }}$ and $\varphi_{\text {dis }}$, and drives the motors of the beam deflection unit.

First, the system model is introduced. The beam path describes the relationship between the laser beam from the mirror to the retroreflector and the rotation angle of the galvanometer scanner. Moreover, the beam path model is a function of the absolute distance between the mirror and the retro-reflector. The beam path model is given as follows:

$G_{B}=\frac{x_{\text {Beam }}}{\varphi}=2 \ell_{\text {rad }}$

The dynamics of the galvanometer scanner can be approximated by a second order differential equation. The model parameters have to be identified using experimental data. The model can be expressed in Laplace space as follows:

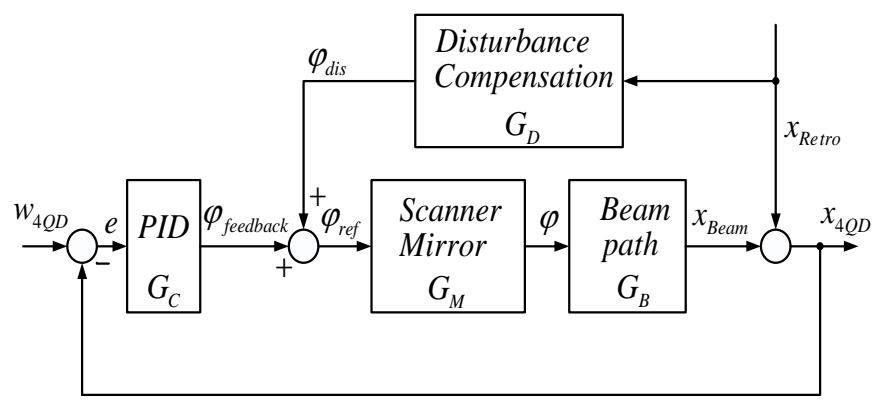

Figure 7. The control scheme. 


$$
G_{M}=\frac{\varphi}{\varphi_{\text {ref }}}=\frac{b_{0}}{a_{0}+a_{1} s+a_{2} s^{2}}
$$

For detailed information regarding the system model the reader is referred to [1].

In the feedback path a digital PID controller is used to suppress the error $e$. The PID parameters are determined using the pole placement method.

The PID controller for single-input and single-output systems can be described in Laplace space as follows:

$G_{C}=\frac{K_{I}+K_{P} s+K_{D} s^{2}}{s}$

The controller affects on the error $e$, which is the difference between the control variable $x_{4 Q D}$ and the set point value $w_{4 Q D}$. The set point of the feedback controller is $w_{4 Q D}=0$.

In the case that the motion of the tracking kinematics is known, the path planning signals of the kinematics can be used to compensate the disturbance $x_{\text {Retro }}$. Hence, the disturbance compensator $G_{D}$ (see Figure 7) has to be taken into account. Its dynamical behaviour can be approximated by a second order system and is expressed in Laplace space:

$$
G_{D}=\frac{\varphi_{\text {dis }}}{X_{\text {Retro }}}=k_{0}+k_{1} s+k_{2} s^{2}
$$

To determine the parameters of the PID controller a transfer function of the whole closed loop shown in Figure 7 has to be found. The output of the transfer function is the control variable $x_{4 O D}$ and the input is the motion of the retroreflector $x_{\text {Retro }}$. Thus the transfer function can be expressed as follows:

$G_{W}=\frac{x_{4 Q D}}{x_{\text {Retro }}}=\frac{1+2 G_{D} G_{B} G_{M}}{1+2 G_{C} G_{B} G_{M}}$

Inserting Equations (3), (4), (5) and (6) in equation (7) yields:

$$
G_{W}=\frac{s / a_{2}\left(s^{2}\left(a_{2}+k_{2} \varepsilon\right)+s\left(a_{1}+k_{1} \varepsilon\right)+\left(a_{0}+k_{0} \varepsilon\right)\right)}{s^{3}+s^{2} \frac{\left(a_{1}+K_{D} \varepsilon\right)}{a_{2}}+s \frac{\left(a_{0}+K_{P} \varepsilon\right)}{a_{2}}+\frac{K_{I} \varepsilon}{a_{2}}}
$$

with:

$$
\varepsilon=4 b_{0} \ell_{\text {rad }}
$$

In Equation (8) the polynomial $A(s)$ represents the numerator and $B(s)$ describes the denominator of the transfer function. The transfer function decreases to zero for $t \rightarrow \infty$, consequently $s \rightarrow 0$ :

$\lim _{s \rightarrow 0} G_{W}=\frac{A(s)}{B(s)}=0$

Equation (10) shows clearly that the proposed model-based controller is able to track the target with a tracking error of zero. The dynamical behaviour of equation (10) depends on the parameters of the denominator $B(s)$ as well as the controller parameters $K_{P}, K_{D}$ and $K_{I}$. To calculate the parameters of the denominator $B(s)$ the following desired polynomial $P(s)$ is used:

$$
P(s)=s^{3}+p_{2} s^{2}+p_{1} s+p_{0}
$$

Then the desired polynomial $P(s)$ is equated with the denominator $B(s)$ of equation (11) and the parameters of the PID controller are calculated as follows:

$$
K_{I}=\frac{p_{0} a_{2}}{4 b_{0} \ell_{\mathrm{rad}}}, K_{P}=\frac{p_{0} a_{2}-a_{0}}{4 b_{0} \ell_{\mathrm{rad}}}, K_{D}=\frac{p_{0} a_{2}-a_{1}}{4 b_{0} \ell_{\mathrm{rad}}}
$$

Equation (12) shows that the parameters $K_{I}, K_{P}$ and $K_{D}$ are not constant and change as a function of the absolute distance
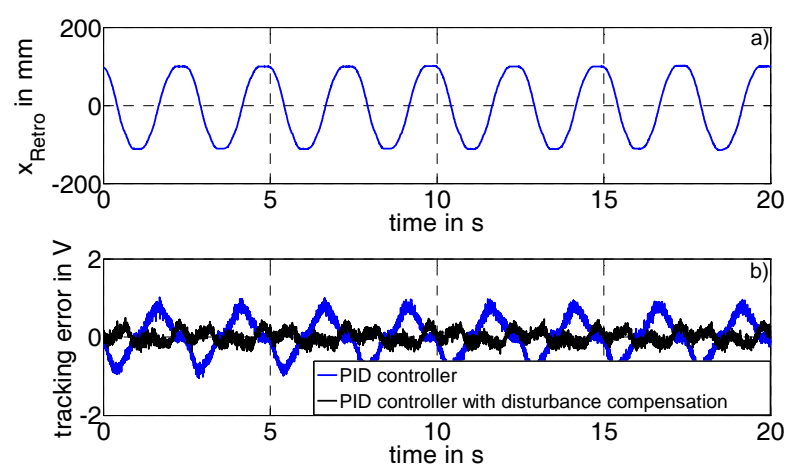

Figure 8 . The sinusoidal motion $f=0.4 \mathrm{~Hz}$ of the tracked kinematics a) and the tracking error $b$ ).

between the laser tracker and the retroreflector. Due to the fact that the interferometers can only measure a relative distance, there is a need to determine the absolute distance. To solve this problem we have developed a triangulation method, which opens the possibility to determine the absolute distance with the precision needed by using at least two tracker modules [2] [3] [4].

The proposed control algorithm was experimentally validated by a kinematical system, which moved along one axis. Figure 8 a shows the sinusoidal motion of the tracked kinematics and Figure $8 \mathrm{~b}$ shows the resulting tracking error of the laser beam. The dynamical set-points of the kinematics were generated by an analytic fourth order path planning algorithm, which is able to plan motions with arbitrary initial and final velocities [10]. The kinematics carried out a sinusoidal motion with amplitude of $100 \mathrm{~mm}$ and a frequency of $0.4 \mathrm{~Hz}$.

Without disturbance compensation the tracking error of the laser beam is approximately $1 \mathrm{~V}$ at peak and a clear phase shift is observable. Using the proposed model-based disturbance compensation the tracking error can be reduced by a factor of 2. Moreover, the phase shift is not noticeable anymore and this shows the effectiveness of the presented model based control approach.

\section{CALCULATION OF THE TCP}

\subsection{Calculation of the TCP for one tracker module}

After the implementation of the required control algorithms one tracker module is able to measure the position of the retroreflector, viz. the TCP position. In the case of one tracker module the determination of the TCP position is carried out using vector analysis. This operation requires a precise geometrical model of the beam path. Figure 9 shows the geometric relation between laser beam and beam deflection system. The laser beam starts from point $P O$ and hits the $\mathrm{x}$-scanner mirror in the point $P 1$. The $\mathrm{x}$-scanner mirror reflects the laser beam to the $y$-scanner mirror at point $P 2$ and then the laser beam is reflected to the retroreflector at the TCP. To calculate the TCP the points $P 0, P 1, P 2$ have to be known in a predefined coordinate system. The proposed coordinate system uses the rotation axes of the beam deflection system as orthogonal axes:

$\overrightarrow{v_{1}}=\left[v_{1 x}, v_{1 y}, v_{1 z}\right]^{T}, \overrightarrow{v_{2}}=\left[v_{2 x}, v_{2 y}, v_{2 z}\right]^{T}$

First, the rotation axis of the $y$-scanner is used as the $x$-axis of the coordinate system. Second, the z-axis is defined by the cross product of the rotation axes, which is perpendicular to the 

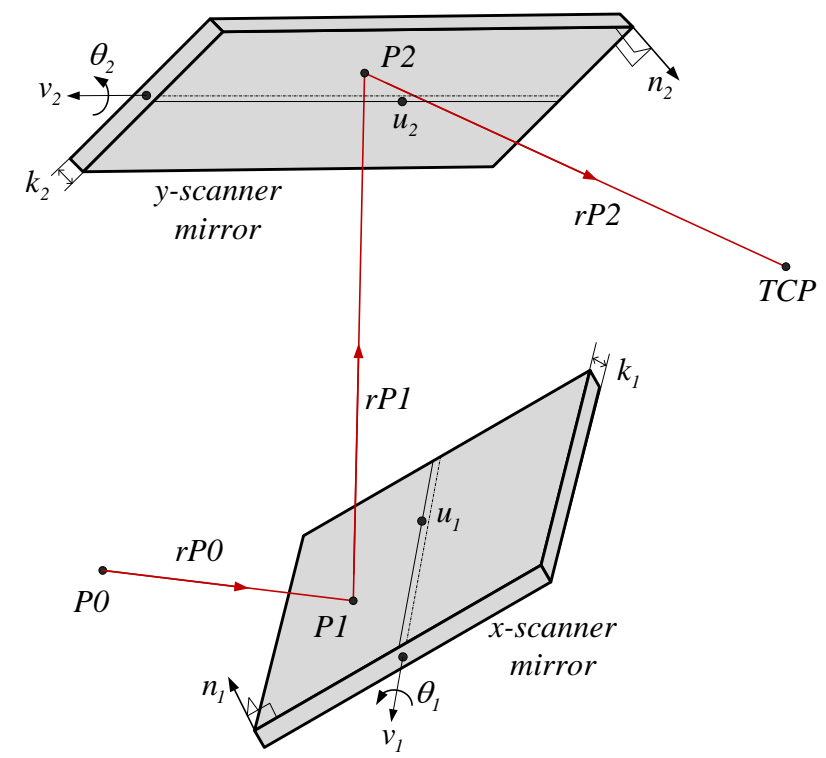

Figure 9. The geometric representation of the beam deflection system.

$x$-axis. Finally, the $y$-axis is the cross product of $x$ - and $z$-axis. All axes are given as follows:

$$
\begin{aligned}
& \vec{x}=\vec{v}_{1} \\
& \vec{z}=\vec{v}_{1} \times \vec{v}_{2} \\
& \vec{y}=\vec{x} \times \vec{z}
\end{aligned}
$$

The laser beam is considered to be a straight line represented by a position and a direction vector. The direction vector after an arbitrary deflection can be computed as follows:

$$
\vec{r}_{1}=\vec{r}_{0}-2\left(\vec{r}_{0} \vec{n}\right) \vec{n}
$$

The vector $r_{0}$ is the direction vector of the laser beam before the deflection and the vector $n$ is the normal vector of the utilized mirror. In the case that the vector $r P 0$ is known, the direction vectors $r P 1, r P 2$ can be calculated using equation (15) (see Figure 9). Furthermore, the direction vectors are used to determine the reflection points P1, P2. The rotating mirror is treated as a plane described by a point on the surface and the according normal vector. The intersections P1 and P2 can be calculated as follows:

$$
\begin{aligned}
& \overrightarrow{P 1}=\overrightarrow{P 0}+\frac{\left(\vec{u}_{1}-\overrightarrow{P 0}\right) \vec{n}_{1}}{\overrightarrow{r P 0} \cdot \vec{n}_{1}} \overrightarrow{r P 0} \\
& \overrightarrow{P 2}=\overrightarrow{P 1}+\frac{\left(\vec{u}_{2}-\overrightarrow{P 1}\right) \vec{n}_{2}}{\overrightarrow{r P 1} \cdot \vec{n}_{2}} \overrightarrow{r P 1}
\end{aligned}
$$

The vectors $n 1, n 2$ are the normal vectors of the mirrors. The vectors $u_{1}$ and $u_{2}$ represent an arbitrary point on every mirror surface. A detailed description of the presented geometric model is shown in [14], [15]. In the case of a moving TCP the points $P 1$ and $P 2$ are variant due to the rotation of the mirrors. A normal vector of the mirror has to be updated online using the rotation axis $v_{i}=\left[v_{i x} v_{i y} v_{i r}\right]$ as well as the rotation angle $\theta$ :

$\tilde{n}_{i}=\boldsymbol{R} \cdot n_{i}, i=1,2$

with

$$
\begin{gathered}
\boldsymbol{R}=\left[\begin{array}{ccc}
v_{i x}{ }^{2} \Psi+C & v_{i x} v_{i y} \Psi-v_{i z} S & v_{i x} v_{i z} \Psi+v_{i y} S \\
v_{i x} v_{i y} \Psi+v_{i z} S & v_{i y}{ }^{2} \Psi+C & v_{i y} v_{i z} \Psi-v_{i x} S \\
v_{i x} v_{i z} \Psi-v_{i y} S & v_{i y} v_{i z} \Psi+v_{i x} S & v_{i z}{ }^{2} \Psi+C
\end{array}\right], \\
\Psi=1-\cos \left(\theta_{i}\right), C=\cos \left(\theta_{i}\right), S=\sin \left(\theta_{i}\right), i=1,2
\end{gathered}
$$

After the determination of the deflection points P1 and P2 the TCP can be calculated by using the straight line with an initial position at point $P 2$ and a direction vector $r P 2$. The TCP is given as follows:

$$
\overrightarrow{T C P}=\overrightarrow{P 2}+\ell \cdot \overrightarrow{r P 2}
$$

The parameter $\ell$ is the radial distance between the y-scanner mirror and the TCP and $\ell$ is the sum of the unknown initial length $\ell_{0}$ and the measured relative distance $\Delta \ell$.

The proposed calculation method of the TCP requires the determination of the unknown system parameters as well as the unknown initial length. After the calibration process the presented geometric model is used to correct the length measurement of the interferometer [14][15].

\subsection{High precision determination of the TCP position using the MLTS}

The TCP position can be calculated by the relative length measurements of all interferometers. Figure 10 shows the four laser trackers $\left(T_{1}, T_{2}, T_{3}\right.$ and $\left.T_{4}\right)$ and the position of the retroreflector $P_{T C P}=[X, Y, Z]^{T}$ in the global Cartesian coordinate system. Every tracker module has three location parameters

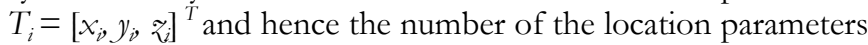
lead to a total of twelve location parameters describing the whole multi-laser tracking system.

To reduce the number of the unknown location parameters from twelve to six and simplify the calculation of the TCP position, the geometric configuration in Figure 10 is chosen. For detailed information regarding the choice of the global coordinate system the reader is referred to [17] [18]. The tracker $T_{1}$ is located in the origin of the coordinate system and has position $[0,0,0]^{T}$. The tracker $T_{2}$ is located in the $x$-axis, the tracker $T_{3}$ is located on the $x-y$-plane and the position of the tracker $T_{4}$ is freely selectable. The parameter $\ell_{i}$ describes the radial distance of the four laser trackers and is defined as follows:

$\ell_{i}=\ell_{0 i}+\Delta \ell_{i}, i=1 \ldots 4$

The parameter $\ell_{0 i}$ represents the offset length (dead zone) after the initialization phase and the interferometer detects the relative radial distance $\Delta \ell_{i}$. If the positions of all trackers and all

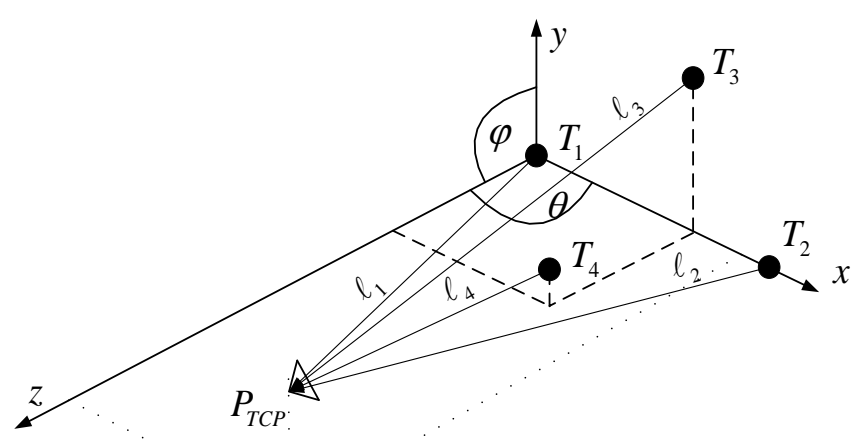

Figure 10. The position configuration of the MLTS. 
distances $\ell_{0 i}$ are known, the TCP position can be determined by an approach called multilateration. by:

The four spherical equations in the Euclidian space are given

$$
\begin{aligned}
& X^{2}+Y^{2}+Z^{2}=\left(\ell_{01}+\Delta \ell_{1}\right)^{2} \\
& \left(X-x_{2}\right)^{2}+Y^{2}+Z^{2}=\left(\ell_{02}+\Delta \ell_{2}\right)^{2} \\
& \left(X-x_{3}\right)^{2}+\left(Y-y_{3}\right)^{2}+Z^{2}=\left(\ell_{03}+\Delta \ell_{3}\right)^{2} \\
& \left(X-x_{4}\right)^{2}+\left(Y-y_{4}\right)^{2}+\left(Z-z_{4}\right)^{2}=\left(\ell_{04}+\Delta \ell_{4}\right)^{2}
\end{aligned}
$$

After the insertion of Equation (22) in Equation (23), Equation (24), Equation (25) and using of the relation $\ell_{i}=\ell_{0 i}+\Delta \ell_{i}$, the following linear system of equations can be defined [17]:

$$
2 \underbrace{\left[\begin{array}{lll}
x_{2} & 0 & 0 \\
x_{3} & y_{3} & 0 \\
x_{4} & y_{4} & z_{4}
\end{array}\right]}_{M}\left[\begin{array}{l}
X \\
Y \\
Z
\end{array}\right]=\left[\begin{array}{c}
\ell_{1}^{2}-\ell_{2}^{2}+x_{2}^{2} \\
\ell_{1}^{2}-\ell_{3}^{2}+x_{3}^{2}+y_{3}^{2} \\
\ell_{1}^{2}-\ell_{4}^{2}+x_{4}^{2}+y_{4}^{2}+z_{4}^{2}
\end{array}\right]
$$

It is possible to calculate the TCP if the matrix $\mathbf{M}$ can be inverted:

$$
\left[\begin{array}{l}
X \\
Y \\
Z
\end{array}\right]=\frac{1}{2} \boldsymbol{M}^{-1}\left[\begin{array}{c}
\ell_{1}^{2}-\ell_{2}^{2}+x_{2}^{2} \\
\ell_{1}^{2}-\ell_{3}^{2}+x_{3}^{2}+y_{3}^{2} \\
\ell_{1}^{2}-\ell_{4}^{2}+x_{4}^{2}+y_{4}^{2}+z_{4}^{2}
\end{array}\right]
$$

The inverted matrix $\mathbf{M}$ is given by:

$$
\boldsymbol{M}^{-1}=\left[\begin{array}{ccc}
\frac{1}{x_{2}} & 0 & 0 \\
-\frac{x_{3}}{x_{2} y_{3}} & \frac{1}{y_{3}} & 0 \\
-\frac{x_{4}}{x_{2} z_{4}}+\frac{x_{3} y_{4}}{x_{2} y_{3} z_{4}} & -\frac{y_{4}}{y_{3} z_{4}} & \frac{1}{z_{4}}
\end{array}\right]
$$

\subsection{Estimation algorithm of the system parameters}

Equation (25) can be used to prove the validity of the calculated TCP position. Therefore, the location parameters $x_{4}, y_{4}$ and $\tau_{4}$ as well as $\ell_{04}$ are needed. The offset length $\ell_{04}$ is calculated by substituting equation (27) in equation (25). The relative distances can be set to zero in order to determine $\ell_{04}$ which is a function of the location and the remaining offset length parameters:

$$
\ell_{04}=f_{\ell_{04}}\left(x_{2}, x_{3}, y_{3}, x_{4}, y_{4}, z_{4}, \ell_{01}, \ell_{02}, \ell_{03}\right)
$$

with $\Delta \ell_{1}=\Delta \ell_{2}=\Delta \ell_{3}=0$

To achieve a simplification the location parameters and the independent offset length parameters can be denoted as follows:

$$
\begin{aligned}
& \underline{p}_{l}=\left[x_{2}, x_{3}, y_{3}, x_{4}, y_{4}, z_{4}\right] \\
& \underline{p}_{o}=\left[\ell_{01}, \ell_{02}, \ell_{03}\right]
\end{aligned}
$$

In order to calculate the TCP position very precisely the objective is the determination of the optimal parameter values. As the location parameters are static they can be estimated offline. For this purpose a pattern-search algorithm is used [23]. That method is rather time-consuming but has to be accomplished only once. In the case that there is no reference position of the kinematics the offset length parameters change with every shift of the initial position of the TCP. The strategy is to identify the location parameters in a previous step with a pattern-search algorithm. Then, the offset length parameters are estimated in real time.

As an optimization method for the offset length parameters the Gauss-Newton approach is applied. The Gauss-Newton approach is a specific modification of the Newton method and convenient for nonlinear problems [20]. The calculation of the Hessian is very complex and requires a lot of computing resources. Hence, a quasi-Newton approach is applied that does not require the calculation of the Hessian.

The objective function is based on equation (25) and is solved for $\Delta \ell_{4}$ :

$$
\Delta \ell_{4}^{c}=f_{\Delta \ell_{4}}\left(\underline{p}_{l}, \underline{p}_{o}, \Delta \ell_{1}, \Delta \ell_{2}, \Delta \ell_{3}\right)
$$

Equation (31) is a function dependent on the offset length as well as the location parameters. The different distances can be interpreted as input signals. Hence, the objective function results in the difference of the calculated and measured distance of tracker $T_{4}$. To apply the estimation algorithm a definite number of measurements is needed leading to the residual function is defined as follows:

$R_{i}=\Delta \ell_{4 i}^{c}-\Delta \ell_{4 i}$

with $i=1 \ldots N$

where $N$ is the index of the measurement data. Since the objective function is clarified the next step is the application of the Gauss-Newton algorithm. Subsequently, the algorithm shall be outlined.

The goal is to minimize the sum of squares of $R_{i}$ :

$\sum_{i=1}^{N} R_{i}^{2} \rightarrow \min$

The Gauss-Newton approach is an iterative process and the current estimated parameters are modified by a correction term $\underline{c}$

$\underline{p}_{k}=\underline{p}_{k-1}-\underline{C}$

with $\underline{c}=\left(\boldsymbol{D}^{T} \cdot \boldsymbol{D}\right) \cdot \boldsymbol{D}^{T} \cdot \underline{r}$

with $\underline{r}=\left[R_{1}, R_{2}, \ldots, R_{N}\right]^{T}$

The Jacobian matrix $\mathbf{D}$ is determined by the derivatives of the residual function Equation (32), i.e. the offset length parameters. Every row correlates with a measurement value. The Jacobian matrix reads as follows:

$$
\mathbf{D}=\left[\begin{array}{ccc}
\frac{\delta R_{1}}{\delta \ell_{01,1}} & \frac{\delta R_{1}}{\delta \ell_{02,1}} & \frac{\delta R_{1}}{\delta \ell_{03,1}} \\
\frac{\delta R_{2}}{\delta \ell_{01,2}} & \frac{\delta R_{2}}{\delta \ell_{02,2}} & \frac{\delta R_{2}}{\delta \ell_{03,2}} \\
\vdots & \vdots & \vdots \\
\frac{\delta R_{n}}{\delta \ell_{01, n}} & \frac{\delta R_{n}}{\delta \ell_{01, n}} & \frac{\delta R_{n}}{\delta \ell_{01, n}}
\end{array}\right]
$$

Due to the fact that the residuum changes continuously with the adjustment of the parameters the Jacobian $\mathbf{D}$ has to be updated in every iteration step. To determine the end of the iteration a criterion is necessary. Here, the norm of the difference of the modified $\left(\underline{p}_{k}\right)$ and the current $\left(\underline{p}_{k-1}\right)$ parameter vector is used. The calculation will be terminated as soon as the norm gets below a defined threshold, is $e$ :

$\left\|\underline{p}_{k}-\underline{p}_{k-1}\right\| \leq e$

The calibration process runs online and automatically. It shall be depicted in the following. 
After the measurement system is activated the control unit handles the initial procedures that include enabling the actuator and sensor units. If the target is localized by all laser trackers, the system is prepared for the calibration procedure. Here, a cube dimensioned motion with $100 \times 100 \times 100 \mathrm{~mm}^{3}$ is executed. This space is marked with 27 grid points and at every point the positioning stage stops for a short period of time. The trajectory is quasi-static because at every grid point several measurement values are taken to generate an average value. After the cube trajectory is finished the measurement data is used to estimate the offset length parameters online. All 27 measurement points compose the residual function $R$ and the Jacobian D. When the termination criterion is reached the parameters are identified. Then, they are written as fixed values in the control system and the multi laser tracker system is ready to measure the motion of the target.

\subsection{Influence of the initial tracker arrangement on the parameter estimation}

After the self-calibration, a precise estimation of all nine system parameters is available. The real values within the simulation are well known. To evaluate the quality of the estimation, the maximum difference between the known and the estimated parameters is calculated:

$Q=\max \left|\vec{p}_{\text {real }}-\vec{p}^{*}\right|$

First investigations have shown that the optimization algorithm is able to converge to the designated minimum. To analyse the influence of noise and local minima, the starting point for optimization is set into an area of $\pm 50 \mathrm{~mm}$ around the real parameters. The measurement noise of the interferometers has a Gaussian distribution with standard deviation of $1 \mu \mathrm{m}$. These assumptions match with the experimental setup.

The choice of the boundary values has significant influence on the accuracy of the self-calibration algorithm. Then, the number of measurement points $(a G)$, the distance between the different trackers among each other $(a T T)$, the size of the working range for the reference points $(a b T C P)$, the distance between the laser trackers and the measurement area (aTTCP) are investigated. In the simulation these configuration values are varied within the following intervals:

$$
\begin{array}{lll}
\text { - } & a G: & {[27,64,125,256]} \\
\text { - } & \text { aTT in }[\mathrm{m}]: & {[0.2,0.3,0.5,0.8]} \\
\text { - } & \text { abTCP in }[\mathrm{m}]: & {[0.3,0.4,0.5,0.6,0.7,0.8]} \\
\text { - } & \text { aTTCP in }[\mathrm{m}]: & {[0.5,0.7,0.9,1.1,1.3,1.5,1.7,1.9]}
\end{array}
$$

For $a G$ and $a T T$ there exist four variations of boundary values, six for $a b T C P$ and eight for aTTCP. The total number of calibration set-ups $N_{C}$ is the product of the configuration variations providing the different maximum combination options. $N_{C}$ is calculated as follows:

$$
N_{C}=a G \cdot a T T \cdot a b T C P \cdot a T T C P=4 \cdot 4 \cdot 6 \cdot 8=768
$$

For every combination the optimization is carried out and the quality $Q$ is determined. The current boundary and the optimization results are stored. For the evaluation the maximum aberration between the estimated parameters and the real parameters is compared with a threshold value of $50 \mu \mathrm{m}$. The combination is valid if the maximum aberration is below the threshold value. In the case that all combinations are valid, a maximum number of valid calibrations $N_{M A X}$ can be defined as the quotient between the total number of the calibrations $N_{C}$ and the number of the used intervals for every boundary:

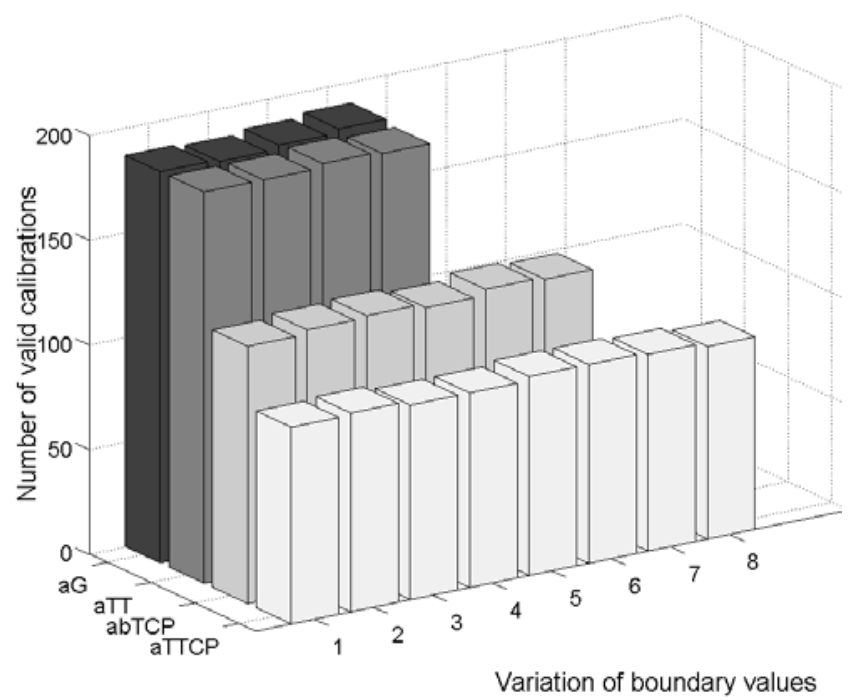

Figure 11. Variation of four boundary values and number of valid calibrations without measurement noise, threshold value $=50 \mu \mathrm{m}$.

$$
\begin{array}{lll}
\text { - } & \mathrm{aG} & \rightarrow \mathrm{N}_{\mathrm{MAX}}=192 \\
\text { - } & \mathrm{aTT} & \rightarrow \mathrm{N}_{\mathrm{MAX}}=192 \\
\text { - } & \text { abTCP } \rightarrow \mathrm{N}_{\mathrm{MAX}}=128 \\
\text { - } & \text { aTTCP } \rightarrow \mathrm{N}_{\mathrm{MAX}}=96
\end{array}
$$

The results of a simulation without measurement noise are presented in Figure 11. The number of valid calibrations is minimal below the maximum number of valid calibrations $N_{M A X}$. It can be stated that the utilized estimation algorithm is appropriate for the selected boundary values.

Figure 12 depicts the simulation results with measurement noise. It can clearly be seen that the choice of the boundaries influences the quality of the parameter estimation.

Only 508 out of 768 calibrations are below the threshold value. The boundary $a T T$ shows the strongest sensitivity regarding a valid calibration. Furthermore, a large distance between the trackers among each other significantly increases the number of the valid calibrations. A step-by-step rise of the aTTCP decreases the number of valid calibrations. In contrast, an increasing $a G$ as well as an increasing $a b T C P$ rises the number of the valid calibrations only slightly.

To show the effect of the boundary values, the threshold

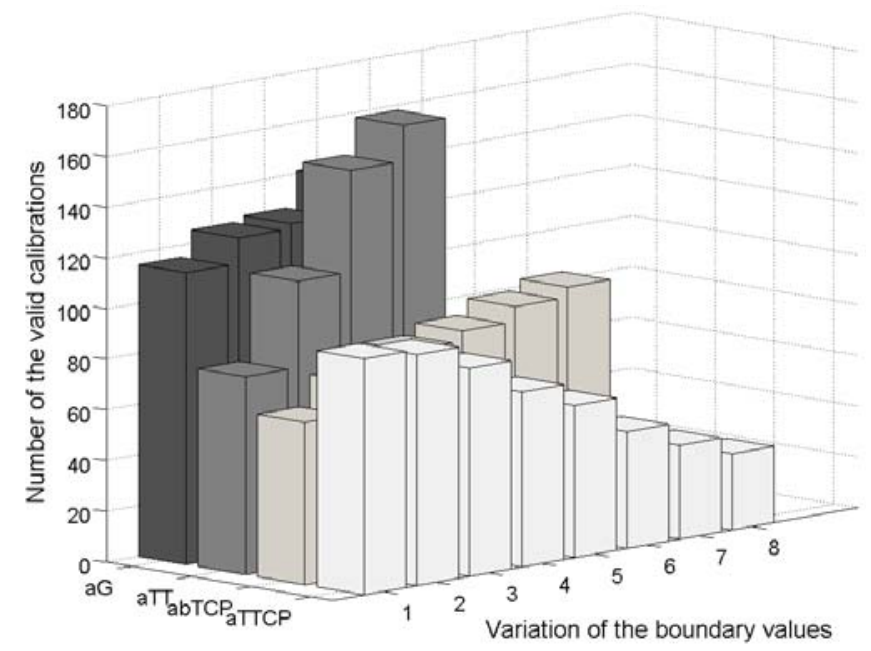

Figure 12. Variation of four boundary values and number of valid calibrations with measurement noise, threshold value $=50 \mu \mathrm{m}$. 


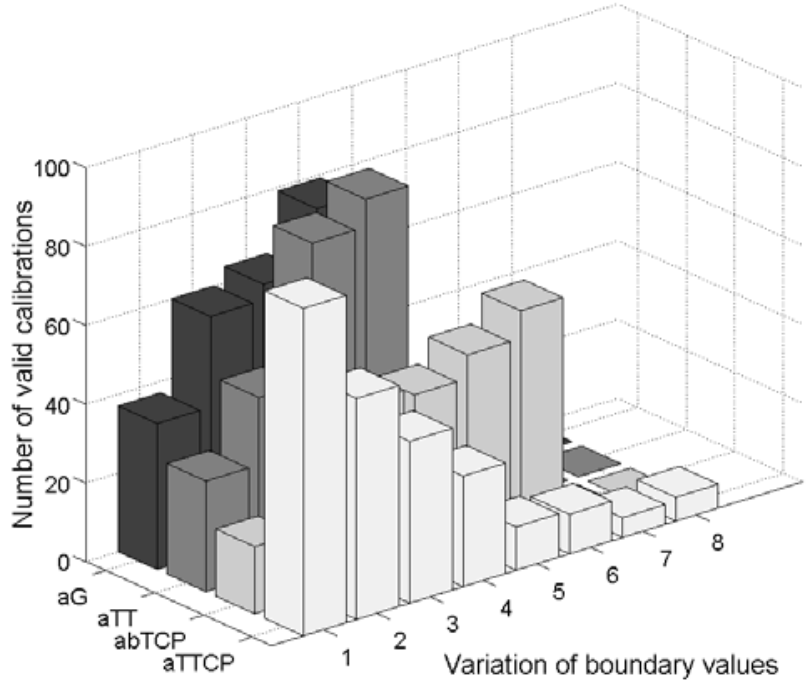

Figure 13. Variation of four boundary values and number of valid calibrations with measurement noise, threshold value $=10 \mu \mathrm{m}$

value is changed in the simulation from $50 \mu \mathrm{m}$ to $10 \mu \mathrm{m}$. In Figure 13 the variation of the boundary values and the related valid calibrations is depicted.

Only 240 of 768 calibrations are below the threshold value. It can be seen that the number of successful optimizations varies extremely with the choice of the boundaries. The number of valid calibrations increases with the number of measurement points $a G$ as well as with an increasing distance between the laser trackers $a T T$. The results further improve with a larger area of reference points.

The best performance for aTTCP is reached for a minimal distance between the trackers and the measurement interval. Further simulations showed that unequally spaced reference points have no negative impact on the estimation error as long as every direction in space is used for measurement. If points only lie in one or two spatial directions, one cannot act on the assumption that all effects of the parameters are gathered. In Table 1 the estimated parameter values for a non-uniform distribution are shown, following the rules mentioned above.

\section{CONCLUSIONS}

In the first part of this contribution we present the developed multi laser tracker system and the required control algorithms allowing to track a highly dynamic moving target. The proposed control algorithms include the model based tracking control, the localization routines for finding the retro-reflector and the communication between the four laser trackers.

In the second part of this work the authors present a method to determine the position of the TCP based on a multilateration algorithm. The key point in the calculation of the TCP is

Table 1. Estimated Parameters [mm].

\begin{tabular}{|c|c|c|c|c|c|}
\hline Parameter & $\ell_{1}$ & $\ell_{2}$ & $\ell_{3}$ & $x_{2}$ & $x_{3}$ \\
\hline Real & 786.8714 & 692.2187 & 706.3521 & 300.0000 & 150.0000 \\
\hline Diff. & 0.0022 & 0.0032 & 0.0029 & 0.0005 & 0.0001 \\
\hline Parameter & $y_{3}$ & $x_{4}$ & $y_{4}$ & $Z_{4}$ & \\
\hline Real & 160.0000 & 150.0000 & 50.0000 & 150.0000 & \\
\hline Diff. & 0.0002 & 0.0005 & 0.0000 & 0.0008 & \\
\hline
\end{tabular}

the determination of nine system parameters. The estimation of these parameters is highly dependent on the initial arrangement of the MTLS. In order to evaluate the sensitivity of the parameter estimation various arrangements of the MLTS were investigated. The presented simulation shows that the choice of the boundary values affects the estimation quality significantly. A sufficiently large distance between laser trackers among each other is required to achieve good results. Furthermore, the reference points should be distributed in a large area and a minimal distance between the laser trackers and the measurement area is recommended. Finally, a large number of measurement points ensures the convergence of the parameter estimation.

\section{ACKNOWLEDGEMENT}

This work was done within the framework of the project "Kompetenzdreieck Optische Mikrosysteme - Spitzenforschung und Innovation in den neuen Ländern", which is supported by the German Ministry of Education and Research (FKZ: 16SV5473). The authors would also like to thank all colleagues, who offered help with the work presented.

\section{REFERENCES}

[1] T. T. Nguyen, Q. T. Nguyen, A. Amthor, C. Ament, "Control of a multi laser tracker system used as a position feedback sensor", Proceedings of the IASTED International conference on Modelling, Identification and Control, AsiaMIC (2010), Phuket, Thailand, pp. 132-137.

[2] T. T. Nguyen, A. Amthor, C. Ament, "Communication of the multi laser tracker system used as position feedback sensor", Sustainable Design, Manufacturing and Engineering Workforce, Education for a Green Future, SPIE Eco-Photonics 2011, Strasbourg, France, 8065-32.

[3] T. T. Nguyen, A. Amthor, C. Ament, "High Precision Laser Tracker System for Contactless Position Measurement”, In: IEEE International Conference on Control System, Computing and Engineering, Proceedings, ICCSCE, Penang, Malaysia, 2011.

[4] T. T. Nguyen, A. Amthor, S. Zschaeck, S. Lambeck, "Model Based Control of a Linear Stage Using a Contactless Optical Sensor System", In: IEEE International Conference on Control System, Computing and Engineering, Proceedings, ICCSCE, Penang, Malaysia, 2011.

[5] T. T. Nguyen, A. Amthor, M. Springer, C. Ament, "Nonlinear Modelling and Identification of a High Precision Laser Tracking System", In: International Conference on Control, Automation and Information Science (ICCAIS), Proceedings, Ho Chi Minh City, Vietnam, 2012.

[6] E. Markweg; T. T. Nguyen; S. Weinberger; C. Ament; M. Hoffmann, "Development of a miniaturized multisensory positioning device for laser dicing technology", Laser in Manufacturing, Physics Procedia Volume 12, Part 2, pp. 387-395, Munich 2011.

[7] K. Lau, R. Hocken, W. Haight, "Robot performance measurements using automatic laser tracking techniques", NBS-Navy NAV/CIM, Workshop on Robot Standards, Detroit, 1986.

[8] K. Lau, R. Hocken, W. Haight, "Automatic laser tracking interferometer system for robot metrology", Precision Engineering, no. 8, pp. 3-8, 1986.

[9] A. Amthor, S. Zschaeck, C. Ament, "High precision position control using an adaptive friction compensation approach", IEEE Transactions on automatic control, Vol. 55, no. 1, January 2010, pp. 274-278.

[10] A. Amthor, J. Werner, A. Lorenz, S. Zschaeck, C. Ament, "Asymmetric Motion Profile Planning for Nanopositioning and Nanomeasuring Machines", Journal of Systems and Control Engineering, vol. 224, no. 1, 2010. 
[11] A. Amthor, S. Zschaeck, C. Ament, "Dynamical Friction Modelling and Adaptive Compensation on the Nanometer Scale", 37th Annual Conference of the IEEE Industrial Electronics Society, Proceedings, pp. 511 - 516, Melbourne, 2011.

[12] S. Zschaeck, A. Amthor, C. Ament, "Decentralized High Precision Motion Control for Nanopositioning and Nanomeasuring Machines", 37th Annual Conference of the IEEE Industrial Electronics Society, Proceedings, pp. 499 - 504, Melbourne, 2011.

[13] J. Wang, Y. Liu, Z. Hu, J. Liang, "Modeling and algorithm of attitude measurement of moving target by laser tracking systems", Optical Engineering, Vol. 42, No. 2, pp. 373-382, 2003.

[14] J.R. Mayer, G.A. Parker, "A portable instrument for 3-D dynamic robot measurements using triangulation and laser tracking”, IEEE Transaction on robotics and automation, Vol. 10, no. 4, pp. 504-516, 1994.

[15] J.R. Mayer, G.M. Cloutier, C. Fortin, "Quadrilateration and measurement site identification with dual scanning axis laser tracking interferometers", Measurement Vol. 20, No.3, pp. 171 187, 1997.

[16] J.R. Mayer, G. A. Parker, "Calibration and assessment of a laser based instrument for robot dynamic measurement", 11 th Trien- nal World Cong. Int. Measurement Confederation (IMEKO), Houston, TX, Oct. 1988, vol.: Theory, Simulation, Calibration, Testing, Education.

[17] T. Takatsuji, Y. Koseki, M. Goto,T. Kurosawa, "Restriction on the arrangement of laser trackers in laser trilateration", Measurement Science \& Technology, no.9, pp. 1357-1359, 1998.

[18] T. Takatsuji, M. Goto, T. Kurosawa, et al, "The first measurement of a three-dimensional coordinate by use of a laser tracking interferometer system based on trilateration", Measurement Science \& Technology, no.9, pp. 38-41, 1998.

[19] Y. Lin, G. Zhang, "The Optimal Arrangement of Four Laser Tracking Interferometers in 3D Coordinate Measuring System Based on Multi-lateration", Int. Symposium on Virtual Environments, Human-Computer Interfaces, and Measurement Systems, Lugano, Switzerland, pp.138-143, 2003.

[20] O. Nelles, Nonlinear System Identification, Springer Verlag, Berlin, 2001.

[21] SIOS Meßtechnik GmbH, http://www.sios.de, 27.11.2013.

[22] Edmund optics, http://www.edmundoptics.com, 27.11.2013.

[23] TheMathworks, http://www.mathworks.de/de/help/gads/patternsearch.html, 27.11.2013. 